\title{
Primary Cancer Prevention by Green Tea, and Tertiary Cancer Prevention by the Combination of Green Tea Catechins and Anticancer Compounds
}

\author{
Hirota Fujiki', Eisaburo Sueoka', Tatsuro Watanabe ${ }^{1}$, Masami Suganuma ${ }^{2}$ \\ ${ }^{1}$ Department of Clinical Laboratory Medicine, Faculty of Medicine, Saga University, Saga, ${ }^{2}$ Graduate School of Science and Engineering, Saitama \\ University, Saitama, Japan
}

\begin{abstract}
Green tea is a daily beverage, a non-oxidized non-fermented product containing at least four green tea catechins. Considering our first results when repeated applications of (-)-epigallocatechin gallate (EGCG) prevented tumor promotion in mouse skin, we have continued to look at green tea as a possible cancer preventive agent. 1) The 10-year prospective cohort study by Drs. K. Nakachi and K. Imai revealed that drinking 10 Japanese-size cups (120 mL/cup) of green tea per day delayed cancer onset in humans by 7.3 years among females and by 3.2 years among males. The delay of cancer onset is of course significant evidence of primary cancer prevention in humans. 2) In collaboration with Dr. H. Moriwaki's group we successfully presented a prototype of tertiary cancer prevention showing that 10 Japanese-size cups of green tea daily, supplemented with tablets of green tea extract (G.T.E), reduced recurrence of colorectal adenomas in polypectomy patients by $51.6 \%$ (from $31 \%$ to 15\%). 3) In 1999, we first reported that the combination of green tea catechins and non-steroidal anti-inflammatory drugs showed synergistic anticancer effects in both in vitro and in vivo experiments, along with elucidation of the mechanism. 4) Further studies by other investigators have revealed that various combinations of EGCG or green tea extract and anticancer compounds inhibit tumor volume in xenograft mouse models implanted with various human cancer cell lines. Green tea is a cancer preventive, and green tea catechins act as synergists with anticancer compounds.
\end{abstract}

(J Cancer Prev 2015;20:1-4)

Key Words: EGCG, GADD153, Cancer onset, Recurrence of colon polyps

\section{INTRODUCTION}

Numerous phytochemicals have been reported to have cancer preventive activity. ${ }^{1}$ Since the study on cancer prevention began in Japan in 1983, we have studied green tea as a cancer preventive at the National Cancer Center Research Institute in Tokyo and Saitama Cancer Center Research Institute. Green tea contains four main tea catechins: $10 \%$ to $15 \%$ (-)-epigallocatechin gallate (EGCG), $6 \%$ to $10 \%$ (-)-epigallocatechin (EGC), $2 \%$ to $3 \%$ (-)-epicatechin gallate (ECG), and $2 \%$ (-)-epicatechin (EC). ${ }^{2}$ EGCG, EGC and ECG have cancer preventive activity, while EC is usually inactive. In 1987, we first reported that repeated applications of EGCG prevented tumor promotion of both 12-Otetradecanoylphorbol13-acetate and okadaic acid in mouse skin initiated with 7,12- dimethylbenz[a]anthracene: EGCG inhibited tumor promotion through both protein kinase $\mathrm{C}$ inactivation, and inhibition of protein phosphatases 1 and $2 \mathrm{~A}^{3.4}$

Numerous investigators since then have reported that EGCG and green tea catechins can prevent carcinogenesis in rodents in a wide-range of target organs; ${ }^{5}$ the systemic effect of EGCG was proved by incorporation of ${ }^{3} \mathrm{H}$-EGCG into various organs in mice. ${ }^{6}$ Moreover, frequent drinking of green tea each day plays a significant role in cancer prevention, based on the result that duplicate administrations of ${ }^{3} \mathrm{H}$-EGCG at 6 hours intervals enhanced incorporation of ${ }^{3}$ H-EGCG 4 to 9 fold in most organs compared with a single administration. ${ }^{6}$

Received January 29, 2015, Revised March 19, 2015, Accepted March 19, 2015

Correspondence to: Hirota Fujiki

Department of Clinical Laboratory Medicine, Faculty of Medicine, Saga University, 5-1-1 Nabeshima, Saga 849-8501, Japan

Tel: +81-92-292-0668, Fax: +81-92-292-0668, E-mail: uv4h-fjk@asahi-net.or.jp

Copyright (C) 2015 Korean Society of Cancer Prevention

(c) This is an Open Access article distributed under the terms of the Creative Commons Attribution Non-Commercial License (http://creativecommons. org/icenses/by-nc/3.0) which permits unrestricted non-commercial use, distribution, and reproduction in any medium, provided the original work is properly cited. 


\section{DELAY OF CANCER ONSET WITH 10 CUPS OF GREEN TEA PER DAY}

In 1986, Drs. Nakachi and Imai at Saitama Cancer Center Research Institute surveyed 8,552 individuals aged over 40 on their living habits, including their daily consumption of green tea. During the 10 years after 1986, a total of 419 cancer patients, 244 males and 175 females, were found. These 419 cancer patients were divided into three groups, based on daily consumption of green tea: under 3 cups, 4 to 9 cups and over 10 cups. Next, the average age at cancer onset was obtained from National Health Insurance receipts. ${ }^{7}$

Cancer onset in female patients who had consumed over 10 cups of green tea per day was 7.3 years later than that of patients who had consumed less than three cups per day, and in male patients who had consumed over 10 cups of green tea per day it was 3.2 years later than that of patients who had consumed less than three cups per day. ${ }^{7}$ The difference between females and males may be partly due to higher tobacco consumption by males. This prospective cohort study resulted in a hugely significant finding: drinking 10 cups of green tea per day results in delay of cancer onset among the general population.

\section{PREVENTION OF COLORECTAL ADENOMA RECURRENCE WITH 10 CUPS OF GREEN TEA PER DAY}

As the multistage carcinogenesis of Vogelstein et al. ${ }^{8}$ indicated, colon polyps are the early stage of colon cancer. In collaboration with Dr. Moriwaki's group at Gifu University, we conducted a double-blind randomized clinical Phase II prevention trial to study recurrence of colorectal adenoma. Patients who had no polyps 12 months after the 1st colonoscopy were divided into two groups: Control group maintained daily consumption of green tea without placebo, and the tablets of green tea extract (G.T.E) group took daily 10 cups of green tea supplemented with G.T.E for 12 months. G.T.E was produced by the Green Tea Laboratory of Saitama Prefecture. The results were exciting: The recurrence rate of the control group was $31 \%$, and that of the G.T.E group was $15 \%$ - as determined by end-point colonoscopy 12 months later 9 - so drinking 10 Japanese-size cups of green tea supplemented with G.T.E reduced recurrence of colorectal adenomas by $51.6 \%$, even when a placebo was not used in prevention trial. ${ }^{9}$ Shin $^{10}$ at Seoul National University presented similar results at the International Conference on the 19th Annual Meeting of Korean Society of Cancer Prevention: The results confirmed prevention of colorectal adenoma recurrence.

\section{ANTICANCER ACTIVITY WITH THE COMBINATION OF EGCG AND NSAIDS}

In 1999, we found that the combination of EGCG and sulindac, or EGCG and celecoxib, synergistically enhanced apoptosis in human lung cancer cell line PC-9 cells 11-fold or 15-fold, whereas EGCG, sulindac or celecoxib alone did not induce any apoptosis. ${ }^{11,12}$

The treatments of multiple intestinal neoplasia (Min) mice with a combination of green tea extract and sulindac synergistically reduced the number of tumors per mouse from 72.3 to 32.0 , a decrease of $55.7 \%$, whereas green tea extract alone or sulindac alone inhibited tumor development to a much lesser degree. $^{13}$

The study of molecular mechanisms involved in synergistic enhancement of anticancer activity revealed that the combination of EGCG and sulindac induced a dramatic up-regulation of two genes, growth arrest and DNA damage-inducible gene 153 (GADD 153), and p21, about 12 fold and 3 fold in PC-9 cells. Whereas treatment with either EGCG or sulindac alone had little effect on gene expression. ${ }^{14}$

\section{SYNERGISTIC ANTICANCER EFFECTS WITH THE COMBINATION IN IN VITRO EXPERIMENTS}

Numerous investigators have reported that the combination of EGCG (green tea catechins) with 36 anticancer compounds (Table 1) enhanced in vitro synergistic anticancer effects in 55 human cancer cell lines (Table 2): EGCG and green tea catechins generally increase the anticancer effects of numerous anticancer compounds in various human cancer cell lines, derived from various cancer tissues. ${ }^{15}$

\section{ANTICANCER ACTIVITY IN XENOGRAFT MOUSE MODELS IMPLANTED USING HUMAN CANCER CELL LINES WITH THE COMBINATION}

The inhibition of tumor volume in 13 xenograft mouse models implanted using human cancer cell lines has been reported by other investigators. Average inhibition of tumor volume by the combination of EGCG, or green tea extract, and 13 tested anticancer compounds was $70.3 \%$, while those by anticancer compounds alone, EGCG alone or vehicle for control were $33.7 \%$, 
Table 1. List of anticancer compounds that have shown synergistic anticancer effects with EGCG, or other green tea catechins

\begin{tabular}{ll}
\hline \multicolumn{1}{c}{ Cancer tissue } & \multicolumn{1}{c}{ Effective anticancer compounds used in experiment } \\
\hline Head, neck and lung & Celecoxib, curcumin, erlotinib, 5-fluorouracil, luteolin, sulindac, tamoxifen \\
Breast & Curcumin, 4-hydroxytamoxifen, raloxifene, resveratrol, tamoxifen, $\gamma$-tocotrienol, tricostatin A \\
Prostate & Bortezomib, docetaxel, doxorubicin, genistein, NS398, paclitaxel, quercetin, resveratrol, sulforaphane \\
Liver & Doxorubicin, 5-fluorouracil \\
Colon & Sodium butyrate, sulforaphane \\
Ovaries & Cisplatin, sulforaphane, trans-palladiums \\
Malignant neuroblastoma & Retinoids (ATRA, 13-cis-RA, 4-HPR), SU5416 \\
Leukemia & Benzyl isothiocyanate, celastrol, curcumin, cytosine arabinoside, $\mathrm{H}_{2} \mathrm{O}_{2}$ \\
Pancreas & Celecoxib, thymoquinone, TRAIL \\
Cervix & Retinoic acid \\
Melanoma & Vorinostat \\
Skin & 3-Deazaneplanocin \\
Stomach & Docetaxel \\
\hline
\end{tabular}

EGCG: (-)-epigallocatechin gallate.

Table 2. List of human cancer cell lines that have shown the synergistic anticancer effects with the combination

\begin{tabular}{ll}
\hline \multicolumn{1}{c}{ Human cancer tissues } & Human cancer cell lines used in experiment \\
\hline Head, neck and lung & A549, ChaGo K-1, H292, H358, H460, H2122, NCI-H460, PC-9, SQCCY1, Tu177, Tu212, YCU-N861, \\
& YCU-H891, 38, 886LN \\
Breast & MDA-MB-231, HS578T, MCF-7 \\
Prostate & ALVA-41, CWR22Rv1, IBC-10a, LNCaP, PC-3, PC-3 AP-1, PC-3ML, PCa-20a, cancer stem cells of PC-3, \\
& RPMI8226 MM \\
Liver & BEL-7404/DOX, Hep3B \\
Colon & HCT-116, HT-29, RKO \\
Ovaries & A2780, A2780 (cisR), SKOV-ip1 (paclitaxel-sensitive), SKOVTR-ip2 (paclitaxel-resistant) \\
Malignant neuroblastoma & SH-SY5Y, SK-N-BE2 \\
Leukemia & B-cell chronic leukemia, HL-60, Jurkat T leukemia, K-562, myelogenous leukemia \\
Pancreas & Colo357, PANC-1, MIA PaCa-2 \\
Cervix & HeLa, TMCC-1 \\
Melanoma & A-375, G-361, Hs-294T \\
Skin & A431, SCC-13 \\
Stomach & BGC-823 \\
\hline
\end{tabular}

$26.5 \%$, or $0 \%$, respectively. ${ }^{15}$ As one specific example, the combinations of EGCG and paclitaxel, and EGCG and docetaxel, completely eliminated tumor development of human prostate cancer cell line PC-3ML in xenograft mouse models. ${ }^{16}$ The amount of EGCG necessary for complete elimination of tumor in mice usually corresponds to 6 to 9 Japanese-size-cups of green tea (1.37-2.05 g EGCG) for humans. ${ }^{15}$ Since mice treated with the combination did not show any toxic effects, the achievement of improved quality of life is anticipated.

\section{CONCLUSION}

It is essential to discuss the effects of EGCG on various cancer stem cells. The combination of EGCG and quercetin synergistically inhibited stem cell characteristics of human prostate cancer cells, ${ }^{17}$ and EGCG alone also inhibited viability of human pancreatic cancer stem cells in primary and secondary spheroids in a dose-dependent manner $(0-60 \mu \mathrm{M}) .{ }^{18}$ Similar results are increasingly reported by other investigators, indicating that EGCG and other green tea catechins target cancer stem cells in numerous human cancer tissues.

\section{ACKNOWLEDGMENTS}

We thank Drs. Kei Nakachi and Kazue Imai at Department of Radiobiology/Molecular Epidemiology, Radiation Effects Research Foundation, Hiroshima, Drs. Hisataka Moriwaki and Masahito Shimizu at Department of Medicine, Gifu University, and Messrs. Yoshiaki Kitaoka, Kenta Nakajima and Dr. Atsushi Takahashi at the Green Tea Laboratory of Saitama Prefectural Agriculture and 
Forestry Research Center, for their fruitful collaborations and stimulating discussion.

\section{CONFLICTS OF INTEREST}

No potential conflicts of interest were disclosed.

\section{REFERENCES}

1. Surh YJ. Cancer chemoprevention with dietary phytochemicals. Nat Rev Cancer 2003;3:768-80.

2. Fujiki H, Okuda T. (-)-Epigallocatechin gallate. Drugs Future 1992; 17:462-4.

3. Yoshizawa S, Horiuchi T, Fujiki H, Yoshida T, Okuda T, Sugimura $\mathrm{T}$. Antitumor promoting activity of (-)-epigallocatechin gallate, the main constituent of "tannin" in green tea. Phytother Res 1987; 1:44-7.

4. Yoshizawa S, Horiuchi T, Suganuma M, Nishiwaki S, Yatsunami J, Okabe S, et al. Penta-O-Galloyl- $\beta$-D-glucose and (-)-epigallocatechin gallate. In: Huang MT, Ho CT, Lee CY, eds. Phenolic compound in food and their effects on health II. Washington, DC, American chemical Society, 1992:316-25.

5. Fujiki $\mathrm{H}$, Suganuma M. Green tea and cancer prevention. Proc Jpn Acad 2002; 78(B):263-70

6. Suganuma M, Okabe S, Oniyama M, Tada Y, Ito H, Fujiki H. Wide distribution of $\left[{ }^{3} \mathrm{H}\right](-)$-epigallocatechin gallate, a cancer preventive tea polyphenol, in mouse tissue. Carcinogenesis 1998;19: 1771-6.

7. Nakachi K, Matsuyama S, Miyake S, Suganuma M, Imai K. Preventive effects of drinking green tea on cancer and cardiovascular disease: epidemiological evidence for multiple targeting prevention. Biofactors 2000;13:49-54.

8. Vogelstein B, Fearon ER, Hamilton SR, Kern SE, Preisinger AC, Leppert $\mathrm{M}$, et al. Genetic alterations during colorectal-tumor development. N Engl J Med 1988;319:525-32.

9. Shimizu M, Fukutomi Y, Ninomiya M, Nagura K, Kato T, Araki H, et al. Green tea extracts for the prevention of metachronous colorectal adenomas: a pilot study. Cancer Epidemiol Biomark Prev 2008; 17:3020-5

10. Shin CM. Chemoprevention in gastrointestinal cancers. International Conference on the 19th Annual Meeting of Korean Society of Cancer Prevention, 2014 December 12-13, Seoul, Korea: 66-7.

11. Suganuma M, Okabe S, Kai Y, Sueoka N, Sueoka E, Fujiki H. Synergistic effects of (-)-epigallocatechin gallate with (-)-epicatechin, sulindac, or tamoxifen on cancer-preventive activity in the human lung cancer cell line PC-9. Cancer Res 1999:59:44-7.

12. Suganuma M, Kurusu M, Suzuki K, Tasaki E, Fujiki H. Green tea polyphenol stimulates cancer preventive effects of celecoxib in human lung cancer cells by upregulation of GADD153 gene. Int J Cancer 2006;119:33-40.

13. Suganuma M, Ohkura Y, Okabe S, Fujiki H. Combination cancer chemoprevention with green tea extract and sulindac shown in intestinal tumor formation in Min mice. J Cancer Res Clin Oncol 2001;127:69-72.

14. Fujiki H, Suganuma M, Imai K, Nakachi K. Green tea: cancer preventive beverage and/or drug. Cancer Lett 2002;188:9-13.

15. Fujiki H, Sueoka E, Watanabe T, Suganuma M. Synergistic enhancement of anticancer effects on numerous human cancer cell lines treated with the combination of EGCG, other green tea catechins, and anticancer compounds [published online ahead of print 28 December, 2014]. J Cancer Res Clin Oncol 2014.

16. Stearns ME, Wang M. Synergistic effects of the green tea extract epigallocatechin-3-gallate and taxane in eradication of malignant human prostate tumors. Transl Oncol 2011;4:147-56.

17. Tang SN, Singh C, Nall D, Meeker D, Shankar S, Srivastava RK. The dietary bioflavonoid quercetin synergizes with epigallocathechin gallate (EGCG) to inhibit prostate cancer stem cell characteristics, invasion, migration and epithelial-mesenchymal transition. J Mol Signal 2010;5:14.

18. Tang SN, Fu J, Nall D, Rodova M, Shankar S, Srivastava RK. Inhibition of sonic hedgehog pathway and pluripotency maintaining factors regulate human pancreatic cancer stem cell characteristics. Int J Cancer 2012;131:30-40. 